\title{
Robust Hybrid Control for Demand Side Management in Islanded Microgrids
}

\author{
Carolina Albea $^{(}{ }^{\text {, }}$ Carlos Bordons $\left.{ }^{(}\right)$, Senior Member, IEEE, and Miguel A. Ridao ${ }^{(}$, Member, IEEE
}

\begin{abstract}
This paper focuses on designing a robust control law to manage the demand response of islanded microgrids composed of shifting and adjusting loads. On one side, Hybrid Dynamical System theory is suited here, because the microgrid model is composed of continuous-time dynamics (the energy stored in the battery and the adjustable loads), and discrete-time dynamics (the shifting loads). On the other side, Multi Agent System theory is used to control the adjusting loads in order to guarantee a consensus between them. Hence, non-uniform convergence of the State of Charge of the battery to a given reference is ensured. Robustness with respect to plug and play of any load and parameter variations is also ensured. Experimental results from a laboratory-scale microgrid validate the approach.
\end{abstract}

Index Terms-Islanded microgrid, demanded side management, hybrid dynamical systems, multi-agent systems.

\section{INTRODUCTION}

A MICROGRID is a system that integrates loads, generators and storage units actuating as a single controllable entity. Microgrids can operate in islanded mode, connected to the distribution network or connected to other microgrids. Microgrids facilitate the penetration of renewable energy sources in the electrical system, reducing energy losses and leading to a new electrical power systems paradigm [1], [2].

Demand Side Management (DSM) is a significant capacity of electrical networks that allows customers to make decisions regarding their energy consumption, and helping operators to reduce the peak load demand and to flatten the load profile. The use of DSM provides profit in many areas, such as:

Manuscript received October 19, 2020; revised January 19, 2021 and June 10, 2021; accepted July 28, 2021. Date of publication August 2, 2021 date of current version October 21, 2021. This work was supported in part by the Agence Nationale de la Recherche (ANR)-France under Grant ANR-18-CE40-0022-01 (Project HISPALIS); in part by the Agencia Estatal de Investigación (AEI)-Spain under Grant PID2019-105890RJI00/AEI/10.13039/501100011033 (Project HYCAM); and in part by the Local Ministry of Economy and Knowledge of Andalusia under Grant US-1265917. Paper no. TSG-01491-2020. (Corresponding author: Carolina Albea.)

Carolina Albea was with CNRS, LAAS, Université de Toulouse, 31400 Toulouse, France. She is now with the Department of System Engineering and Automatic Control, Universidad de Sevilla, 41092 Seville, Spain (e-mail: calbea@us.es)

Carlos Bordons is with the Department of System Engineering and Automatic Control and the Laboratory of Engineering for Energy and Environmental Sustainability, Universidad de Sevilla, 41092 Seville, Spain (e-mail: bordons@us.es).

Miguel A. Ridao is with the Department of System Engineering and Automatic Control, Universidad de Sevilla, 41092 Seville, Spain (e-mail: miguelridao@us.es).

Color versions of one or more figures in this article are available at https://doi.org/10.1109/TSG.2021.3101875.

Digital Object Identifier 10.1109/TSG.2021.3101875 improvement in the efficiency of the system, security of supply and reduction in overall operational costs and environmental impact. On the other hand, the microgrid control problem becomes more complex since new degrees of freedom appear. While DSM was utility-driven in the past, it is moving towards a customer-driven activity in the near future [3] and it can be used in microgrids which are not connected to the distribution network.

In DSM framework, Demand Response (DR) refers to the actions taken by customers that use information (mainly prices) to adjust their loads. The concept of DR encompasses the discretionary changes by consumers of their usual consumption patterns in response to external conditions and has attracted the attention of many researchers [4]. This kind of schemes can be used to avoid undesirable peaks in the demand curve that take place in some time periods along the day, resulting in a more beneficial rearrangement [5]. The same concept can be used inside a microgrid, where the loads take the role played by customers in the electrical network. In this way, the different loads in a microgrid can be adjusted in order to try to adapt the demand to the available generation, allowing the operation of the microgrid even in low-generation situations. Although DSM is a general concept and DR is related to the control actions taken on the load side, in many occasions the terms are used indistinctly. In microgrids, a responsive and controllable demand behavior can compensate the volatility introduced by intermittent resources on the generation side [6]. In this way, some loads can be adjusted (both in amplitude or in connection times) to contribute to fulfil microgrid operation objectives. This can be achieved by the introduction of new manipulated variables (both continuous and binary) in the problem formulation, which makes the control problem more complex.

In a microgrid, loads can be manipulated up to a certain point. There are some critical loads whose demand must always be met. These uncontrollable loads have to be operated at a certain power and a certain time period that cannot be deferred. But, on the other hand, there exist controllable loads whose total consumption or occupied time duration can be modified, such as Electric Vehicles (EV) or Heating, Ventilating and Air-Conditioning (HVAC). Then, some loads can be reduced, shed or postponed during lack of supply or emergency situations or just to optimize the operation of the microgrid. Controllable loads can be classified as: i) deferable/shiftable if their connection can be stopped, restarted or shifted to other time slots, ii) adjustable/curtaible/variable if their consumption can be adjusted to a lower level if 
necessary [7]. However reducing consumption or shifting the load to some other points in time can affect the normal operation of the load, so a certain penalty might be associated to load variation/shifting when implementing the DR procedure and, possibly, also a priority of disconnection of the different loads.

Two main methods can be used for demand management in a microgrid:

- Shifting: this strategy considers the shifting of certain amounts of energy demand from some time periods to others, with lower expected demand, either in response to price signals or to generation forecast. The Energy Management System (EMS) has flexibility to defer some energy packets, but the total amount of power required by the load must be fulfilled for a certain period.

- Curtailment/Shedding/Variation: this strategy consists of adjusting the magnitude of the power that can be demanded by loads when necessary. Accordingly, considering that loads have a certain degree of manipulation, the demanded power can be reduced during certain times in order to improve the operation of the microgrid or during contingencies. A maximum allowable level of curtailment must be specified for each load.

Both mechanisms can be used in grid-connected or in islanded mode in order to improve the economic benefit. However, in the case of islanded mode they can be crucial, since the grid is not available to supply the loads in case of generation deficit. In this case, the required amount of curtailed load must be chosen according to the forecasted deficit and the available storage.

There are several research works that address DR in smart grids or microgrids. The paper [5] presents a strategy based on a load-shifting technique formulated as a minimization problem and solved with a heuristic-based evolutionary algorithm. Another load-shifting application is done in [8] using Genetic Algorithms, minimizing the combination of generation cost and the inconvenience caused to the customer, which is modelled as a polynomial function of the shifting time. A scenario-based stochastic optimization approach is developed in [9] for real-time price-based DR management of residential appliances, which can be embedded into smart meters, considering time-varying electricity price uncertainties. A multiobjective optimization method and a Linear Matrix Inequality (LMI) approach are used in a case study of three microgrids connected to the grid in [7]. The minimization of the operation cost and the inconvenience caused due to shifting or curtailment of loads is a multiobjective optimization problem that may include binary variables associated to the connection/disconnection of loads. Thus, a mixed integer optimization problem must be solved. This can be done in an open-loop fashion or using feedback as done in Model Predictive Control (MPC) [10]. A DR method based on MPC is proposed in [11] considering constraints on the peak shift effect, and an event-triggered mechanism using aggregates. A strategy based on MPC to manage the energy resources of a residential microgrid using load curtailment, that promotes short term reduction of electricity demand in pre-defined hours is presented in [12].
In this work, a DR is proposed here for active power balancing islanded microgrids. The DR is dealt with both shifting and adjusting loads, that can model any demand, by using the Hybrid Dynamical System (HDS) theory given in [13]. On one hand, a control loop manages the shifting loads, through an optimization problem, which looks for leading the State of Charge (SOC) of the battery to a neighborhood of a given reference. On the other hand, another control loop drives the adjustable loads, by using Multi-Agent System (MAS) theory [14], achieving the convergence of these adjustable loads to any leader-follower consensus [15] with the aim at reducing the mentioned neighborhood. This approach guarantees a maximum of supplied loads such that the demand follows the generation. Some properties of the system are revealed, as the characteristic of a non-uniform convergence, and even, the scalability and robustness with respect to any parameter variation (e.g., any adjustable load, which is required to be constant) or a plug-and-play event.

MAS and HDS theory have already been employed to control microgrids. Indeed, a hybrid cascade control algorithm was designed in [16]. Besides this, in [17], a multi agent algorithm based on a hybrid system was considered. A control law focussed on HDS and MAS theory, ensuring stability and robustness is proposed in [18]. These mentioned control works do not deal with a DR. Likewise, [19]-[21] provide a DR in microgrids by applying multi agent system theory. However, these references only consider shifting loads or adjusting loads, without dealing with both at the same time. Moreover, in [22], a DR algorithm based on hybrid multi-agent theory for microgrids was presented, but without stability and robustness guaranties.

Different to many previous works, a DR mechanism that considers both kind of loads (shiftable and adjustable), which models any demand, is proposed here by using HDS and MAS theory. In particular, [5], [8], [9] deal with DR just for shiftable or adjustable loads. References [10]-[12] do not model the demand, as we do here with a discrete-time and continuoustime dynamics (shiftable and adjustable loads, respectively). Moreover, in [5], [7]-[12] is not given stability properties as the non-uniform convergence property given here. Moreover, focusing on the applied theory, HDS and MAS, we mention the works [16]-[18] that do not consider a DR as control problem and [19]-[22] that do not provide stability and robustness guaranties. Then, we highlight that HDS and MAS theory allow to model discrete-time and continuous-time dynamics and ensure stability and robustness of a DR problem.

The rest of the paper is organized as follows: Section II presents the considered islanded microgrid and states the problem. Section III provides some preliminaries definitions. The proposed control is given in Section IV, which is validated through experiment results Section V. A conclusion section closes the paper.

Notation: Throughout the paper $\mathbb{N}$ denotes the set of natural numbers and $\mathbb{R}$ the set of real numbers, $\mathbb{R}^{n}$ the n-dimensional Euclidean space and $\mathbb{R}^{n \times m}$ the set of all real $n \times m$ matrices. The set of non-negative real numbers is denoted by $\mathbb{R}_{\geq 0}$. $0_{n \times m}$ and $1_{n \times m}$ are the zero and ones matrices, respectively, of $n \times m$ dimension. $I_{n}$ is the identity matrix of dimension $n$. 
$\operatorname{diag}\left\{a_{1}, a_{2}, \ldots, a_{n}\right\}$ is a diagonal matrix whose elements are $a_{1}, a_{2}, \ldots, a_{n} \cdot \operatorname{rank}(M)$ denotes the rank of the matrix $M . x^{\top}$ means the transpose vector of $x$ and $x^{+}$represents the value of $x$ after an instantaneous change.

\section{System Description AND PROBlem Formulation}

An islanded microgrid is a system composed of sources, loads and batteries interconnected by a bus line. Microgrids can have several renewable sources as well as different ways of energy storage. The methodology presented in this paper can be applied to any microgrid but, for the sake of simplicity, a microgrid with photovoltaic generation and hydrid storage by means of electricity (batteries and ultracapacitor) and hydrogen (electrolyzer, fuel cell and hydrogen tanks) will be considered. The different powers flows are:

$$
\begin{array}{ll}
P_{b a t} & \text { Battery power. } \\
P_{d} & \text { Demanded power. } \\
P_{g e n} & \text { Netted generated power. } \\
P_{c l, n} & \text { Power of the } n \text {-constant power load. } \\
P_{v l, i} & \text { Power of the } i \text {-variable power load. }
\end{array}
$$

This microgrid presents a set of Constant Power Loads (CPLs) and Variable Power Loads (VPLs), that means, loads whose power is fixed or can change, respectively. Notice that $P_{g e n}$ represents the netted generated power including some power losses, which cannot be neglected. Moreover, the microgrid power balance equation can be written as:

$$
\begin{aligned}
P_{d}(\sigma, t) & =\alpha P_{v l}+\gamma_{\sigma} P_{c l}(t) \\
P_{b a t}(\sigma, t) & =P_{d}(\sigma, t)-P_{g e n}(t)
\end{aligned}
$$

where $P_{v l}(t)=\left[P_{v l, 1}(t), P_{v l, 2}(t), \ldots, P_{v l, i}(t), \ldots, P_{v l, \mathcal{I}}(t)\right]^{\top} \in$ $\mathbb{R}_{\geq 0}^{\mathcal{I}}$ is a vector composed of the powers of the $\mathcal{I}$ VPLs, $\alpha \in$ $\{0,1\}^{1 \times \mathcal{I}}$ is an exogenous vector that gathers whether the $i$ VPL is connected $(\alpha(i)=1)$ or disconnected $(\alpha(i)=0)$, $P_{c l}=\left[P_{c l, 1}, P_{c l, 2}, \ldots, P_{c l, n}, \ldots, P_{c l, N}\right]^{\top} \in \mathbb{R}_{\geq 0}^{N}$ is a vector composed of the power of the $N$ CPLs and $\gamma_{\sigma} \in\{0,1\}^{1 \times N}$ is an input vector with $\sigma \in \mathcal{N}:=\left\{1,2, \ldots, 2^{N}\right\}$, which means that the load $n$-CPL is connected $\left(\gamma_{\sigma}(n)=1\right)$ or disconnected $\left(\gamma_{\sigma}(n)=0\right)$.

By some abuse of notation in the different powers, the dynamic in the SOC, which provides the level of energy in the storage unit, is given by

$$
\begin{array}{r}
\dot{x}=-\eta\left(P_{b a t}\right) \frac{P_{b a t}(\sigma, t)}{C_{\max }} \\
\eta\left(P_{b a t}\right)= \begin{cases}1 / \eta, & \text { if } P_{b a t} \geq 0 \\
\eta, & \text { if } P_{b a t}<0\end{cases}
\end{array}
$$

where for readability, one adopts the notation $x=$ SOC. Likewise, the maximum capacity of the battery in energy units is $C_{\max }$ and the efficient coefficient of storage is $\eta$, which takes two different values, if the battery is discharging $(1 / \eta)$ or charging $(\eta)$.

The battery and the $i$-VPLs are designed such that

$$
\mathrm{SOC}_{m} \leq x \leq \mathrm{SOC}_{M},
$$

being $\mathrm{SOC}_{m}$ and $\mathrm{SOC}_{M}$ the lower and upper bounds of the $\mathrm{SOC}$, respectively, and

$$
P_{m, i} \leq P_{v l, i} \leq P_{M, i},
$$

being $P_{m, i}$ and $P_{M, i}$ the lower and upper bounds respectively, for each $i$-VPL. This saturated model of loads can represent systems as EV charge or heating systems without violating temperature constraints, for instance.

These conditions, (3) and (4), mean that the variables, $x$ and $P_{v l, i}$ are saturated by an upper and lower bounds. Hence, we define

$$
\begin{aligned}
x_{s} & :=\operatorname{sat}\left(x, \mathrm{SOC}_{m}, \mathrm{SOC}_{M}\right), \\
P_{v l, s}:= & {\left[\operatorname{sat}\left(P_{v l, 1}, P_{m, 1}, P_{M, 1}\right), \operatorname{sat}\left(P_{v l, 2}, P_{m, 2}, P_{M, 2}\right), \ldots,\right.} \\
& \left.\operatorname{sat}\left(P_{v l, \mathcal{I}}, P_{m, \mathcal{I}}, P_{M, \mathcal{I}}\right)\right]^{\top},
\end{aligned}
$$

where $\operatorname{sat}\left(s, s_{m}, s_{M}\right)=\max \left\{\min \left\{s, s_{M}\right\}, s_{m}\right\}$ leads to saturate the variable $s$ such that $s$ flows in $\left[s_{m}, s_{M}\right]$. In the sequel, we will adopt, with abuse of notation, $x$ and $P_{v l}$, instead of, $x_{s}$ and $P_{v l, s}$.

Likewise,

$$
P_{v l}:=P_{v l}^{n s}+P_{v l}^{s}
$$

with

$$
\begin{aligned}
P_{v l}^{s} & :=\left[P_{v l, 1}^{s}, P_{v l, 2}^{s}, \ldots, P_{v l, i}^{s}\right]^{\top} \\
P_{v l}^{n s} & :=P_{v l}-P_{v l}^{s},
\end{aligned}
$$

being $P_{v l, i}^{s} \in\left\{0, P_{m, i}, P_{M, i}\right\}$, i.e., each component of $P_{v l}^{s}$ is either the upper and lower bound of $P_{v l, i}$ if the $i$-VPL is saturated, or 0 if the $i$-VPL is non-saturated. Besides, $P_{v l}^{n s}$ is a vector composed of either the $P_{v l, i}$ flowing in $\left[P_{m, i}, P_{M, i}\right]$, if the $i$-VPL is non-saturated, or 0 if the $i$-VPL is saturated.

Moreover, the following conditions define a scenario characterized by a generated power that can supply some loads without getting empty or full the battery.

Definition 1 (Balanced-SOC Mode): A microgrid defined by Eq. (1) and constrained by

$$
\begin{aligned}
& 1_{1, N} P_{c l}+\sum_{i=1}^{\mathcal{I}} P_{M, i} \geq P_{\text {gen }} \\
& 0_{1, N} P_{c l}+\sum_{i=1}^{\mathcal{I}} P_{m, i} \leq P_{g e n} .
\end{aligned}
$$

describes a functioning mode where the generated power can supply the demanded one, such that $\dot{x}$ can go to zero.

This work is oriented to provide control laws to an islanded microgrid working in both: a functioning mode that satisfies conditions (10) and (11), and a microgrid that does not fulfil these conditions.

In the sequel, one can consider $P_{v l, i}^{n s}(t)$ as the variable to be controlled such that all $P_{v l, i}^{n s}(t)$ converge to a consensus from a leader-follower consensus algorithm, through a distributed control. That means there is, at least, a VPL, with the role of leader, which provides the reference for the others $P_{v l, i}^{n s}(t)$ as followers, leading the variable $x$ to any objective. Moreover, this consensus algorithm provides robustness with respect to any parameter variation or plug-and-play mechanism given by $\alpha$, that is, robustness with respect to a failure or connection/disconnection of any adjustable load, VPL. Moreover, $x$ will be also controlled by the variable input $\sigma$, which decides the connected CPLs. These $N$-CPLs will have a priority level 
to be connected to the microgrid, given by the following weight vector: $\beta=\left[\beta_{1}, \beta_{2}, \ldots, \beta_{N}\right]$. In particular, the controlled objective is that $x$ converges to a neighborhood of $x_{r, k}$, which is a sampled reference that follows the variation of $P_{\text {gen }, k}$ that is, it seems reasonable that the higher solar generation, the bigger SOC reference and reciprocally. Indeed, the reference is selected as follows

$$
\begin{aligned}
\chi\left(P_{\text {gen }, k}\right) & =\frac{P_{\text {gen }, k}-P_{\text {gen }, a v}^{e s}}{2 P_{\text {gen,amp }}^{e s}} K_{x_{r}}+\frac{\mathrm{SOC}_{M}+\mathrm{SOC}_{m}}{2} \\
x_{r, k} & =\operatorname{sat}\left(\chi\left(P_{\text {gen }, k}\right), \mathrm{SOC}_{m}, \mathrm{SOC}_{M}\right),
\end{aligned}
$$

where $P_{g e n, k}=P_{g e n}\left(t_{k}\right)$ is an exogenous signal sampled with a sample interval $T_{s}=t_{k+1}-t_{k}$, with $k \in \mathbb{N}$. $P_{\text {gen,av }}^{e s}$ and $P_{\text {gen,amp }}^{\text {es }}$ define the average and amplitude of the estimated generated power, respectively. The SOC response will depend on this suited estimation, but is not the scope of this work. Note that the reference is composed of a bias given by $\frac{\mathrm{SOC}_{M}+\mathrm{SOC}_{m}}{2}$ in order to have enough amplitude for the SOC response and an offset defined by $\frac{P_{g e n, k}-P_{g e n, a v}^{e s}}{2 P_{\text {gen,amp }}^{e s}} K_{x_{r}}$ in order to make better use of the microgrid. Finally, $K_{x_{r}} \in(0,1]$ is a constant parameter that decides the offset, i.e., how the variation of $P_{g e n, k}$ will be taken into account by the reference. Note that if $K_{x_{r}}$ is close to 0 , the offset is 0 and $x_{r, k}$ is the bias $\frac{\mathrm{SOC}_{M}+\mathrm{SOC}_{m}}{2}$. Likewise, if $K_{x_{r}}$ is close to 1 the offset is different to 0 , i.e., when $P_{g e n, k}$ is close to its maximum (minimum) value, $x_{r, k}$ will be near $\mathrm{SOC}_{M}\left(\mathrm{SOC}_{m}\right)$ and when $P_{g e n, k}$ is approximately $P_{g e n, a v}^{e s}, x_{r, k}$ will be close to $\frac{\mathrm{SOC}_{M}+\mathrm{SOC}_{m}}{2}$. Likewise, it is necessary to avoid a Zeno behavior, guaranteeing a minimum dwell time, $T$, for the connected CPLs.

After this system description, the problem can be stated.

Problem 1: The objective is to ensure a DSM of Eq. (1) for a balanced $S O C$, through a hybrid formulation of the system with two control levels.

1) A closed loop managing the connected loads ensuring that the solutions to Eq. (1) converge to the set

$$
\mathcal{A}:=\left\{\left(x, P_{v l}, \sigma, \tau\right):\left|x-x_{r, k}\right|<\varepsilon,\right\},
$$

where $x_{r, k}$ is a sampled reference and $\tau$ is a variable that represents a timer. This reference is computed from the microgrid capacities and the measured $P_{\text {gen }, k}$. Moreover, $\varepsilon$ is a parameter that describes the error given by the CPLs.

2) Another closed loop that establishes a consensus of all $P_{v l, i}^{n s}$, under a leader-follower criteria, such that $\alpha P_{v l}+$ $\gamma_{\sigma} P_{c l}(t)$ converges to $P_{g e n, k}$. This fact implies that $x$ converges to $x_{r, k}$, ensuring robustness of the distributed system with respect to any plug-and-play mechanism or parameter variation.

Note that the problem deals with a shared-load management, providing a scalable and robust controlled system. The first problem objective is to control the CPLs such that $x$ converges to a neighborhood of $x_{r, k},\left|x-x_{r, k}\right|<\varepsilon$, and then, the second problem objective looks for controlling the connected and nonsaturated VPLs to guarantee the convergence of $x$ to $x_{r, k}$.

The robustness guarantees mentioned in item 2), take into account any $P_{v l, i}$ that for any reason needs to be constant.

\section{PRELIMINARIES}

Highlighting that the microgrid is composed of $i \in \mathcal{I}$ VPLs, the idea is to control locally every one of these $i$-subsystems, in such a way that the system enjoys of suited convergence and robustness properties. The next definition about the Laplacian matrix is introduced to define a compact hybrid system that gathers all dynamics.

Definition 2: The Laplacian matrix represents the undirected graph $\mathcal{G}(\mathfrak{L}, \mathcal{E})$, being $\mathfrak{L}=\{1,2, . ., \mathcal{I}\}$ the set of nodes and $\mathcal{E} \subseteq \mathfrak{L} \times \mathfrak{L}$ the set of interconnections between agents. The neighbors of agent $i$ are denoted by $\mathfrak{N}_{i}(\alpha)=\{h \in$ $\left.\mathfrak{L}:(i, h) \alpha_{i} \alpha_{h} \in \mathcal{E}\right\}$. The graph Laplacian of the network is given by $L(\alpha)=\left[l_{i h}(\alpha)\right]$ with

$$
l_{i h}(\alpha):\left\{\begin{array}{lll}
-\alpha_{i} \alpha_{h}, & \text { if } \quad i \in \mathfrak{N}_{i} \\
\left|\mathfrak{N}_{i}(\alpha)\right|, & \text { if } \quad i=h,
\end{array}\right.
$$

where, $\left|\mathfrak{N}_{i}(\alpha)\right|$ denotes the number of neighbors of node $i$.

Note that the Laplacian matrix $L(\alpha)$ is a symmetric positive semi-definite matrix [23].

Moreover some definitions about HDS theory given in [13] are reviewed here.

Definition 3 [13]: The dynamical behavior described by differential equation $(\dot{x}=f(x))$ or inclusion $(\dot{x} \in f(x))$ is referred as flow.

Definition 4 [13]: The dynamical behavior described by difference equation $\left(x^{+}=g(x)\right)$ or inclusion $\left(x^{+} \in g(x)\right)$ is referred as jump.

Definition 5 [13]: A hybrid system $\mathcal{H}=(\mathcal{C}, f, \mathcal{D}, g)$ is defined as follows:

$$
\mathcal{H}: \begin{cases}\dot{x} \in f, & x \in \mathcal{C} \\ x^{+} \in g, & x \in \mathcal{D},\end{cases}
$$

where $f$ and $g$ represent the flow and jump maps, respectively, and $\mathcal{C}$ and $\mathcal{D}$ represent the flow and jump sets, respectively. In other words, the state $x$ can change according the differential inclusion $\dot{x} \in f$ and difference inclusion, $\dot{x} \in g$, while in the flow set, $\mathcal{C}$ or jump set, $\mathcal{D}$, respectively. Note that an equation is a particular case of an inclusion.

Definition 6 [13, Definition 2.3]: A subset $\operatorname{dom} \zeta \subset$ $\mathbb{R}_{\geq 0} \times \mathbb{N}$ is a compact hybrid time domain if dom $\zeta:=\bigcup_{k=0}^{J-1}\left(\left[t_{k}, t_{k+1}, k\right]\right)$ for some sequence of times $0 \leq t_{0} \leq$ $t_{1} \leq t_{2} \leq \cdots \leq t_{J}$.

Definition $\overline{7}$ [13]: Hybrid arcs $\phi(t, k)$ are solutions to a hybrid system $(\mathcal{C}, f, \mathcal{D}, g)$, i.e., for each jump $k \in \mathbb{N}$, the function $t \mapsto \phi(t, k)$ is locally absolutely continuous on the interval $I_{k}=t:(t, k) \in \operatorname{dom}(\zeta)$, being $\operatorname{dom}(\zeta)$ the hybrid time domain defined in [13, Definition 2.3].

\section{HYBRID CONTROL}

Let us adopt here the paradigm given in [13], since the system is composed of continuous-time dynamics, ( $x$ and $P_{v l, i}$ for all $i$ loads) and discrete-time dynamics, $(\alpha)$.

The first problem is focussed on proposing a solution for item 1 of Problem 1. To this end, one can define the time of the battery to achieve $x_{r, k}$ :

$$
\mathcal{T}(x, \sigma):=\frac{x-x_{r, k}}{\eta\left(P_{b a t}\right) \frac{P_{b a t}(\sigma, t)}{C_{\max }}} .
$$


Note, this definition means that $\mathcal{T}(x, \sigma)$ is the elapsed time when $x$ reaches $x_{r, k}$ if the battery is getting empty as well as getting full. However $P_{b a t}(\sigma, t)=0$ means that $\mathcal{T}=\infty$. Indeed, in this situation $x$ can flows forever, without reaching any bound of the battery.

Now, one can define the cost function which is desirable to minimize:

$$
J(x, \sigma):=\frac{K}{\mathcal{T}(x, \sigma)}-\beta \gamma_{\sigma},
$$

where $K>0$ is a weight constant. It is worth noting that this cost function penalizes both a fast battery time of $x$ to achieve, $x_{r, k}$, and a higher number of disconnected CPLs. The last goal is related with the load priorities, given by the weight vector, $\beta$, which minimizes the penalties induced by switching off any CPL. The objective about minimizing the convergence time of $x$ to a neighborhood of $x_{r, k}$ may seem contradictory, because it is usual to minimize system responses. However, in a microgrid, it is not desirable that the battery gets neither empty, nor full very fast. In particular, if one considers a high $x$ and a low $P_{g e n, k}$, which implies a low $x_{r, k}$, it is not desirable that $x$ converges to the low level $x_{r, k}$ very fast, but that the battery gets empty slowly, maximizing its response time.

The cost function $J(x, \sigma)$ is updated in each jump, then we can manage the jumps establishing that no jump occurs whereas $J\left(x, \sigma^{+}\right)>J(x, \sigma)$ or a minimum dwell time, $T$, between two consecutive switching of $\sigma$, has not elapsed yet. This condition will be implemented in the flow and jump sets, below. Likewise, the control law will be encoded by a condition that improves the issues that induce the system to jump. Then, let us select the index $j$ as follows:

$$
j^{*}:=\underset{j}{\operatorname{argmin}}\left(\frac{K}{\mathcal{T}(x, j)}-\beta \gamma_{j}\right) .
$$

This argmin function looks for changing the functioning mode of each CPL: connected, disconnected; minimizing the $x$ slope in order to obtain a smooth SOC evolution to reduce the battery degradation as well as maximizing the number of connected loads. Note that these objectives are opposite. Nevertheless, the first one is weighted by $K$. Indeed, as $K$ is larger, it will be more important to reach a reduced $x$ slope in order to induce that $x$ converges slower to $\mathcal{A}$.

Then, it is possible to define the hybrid model scheme according to Definition 5,

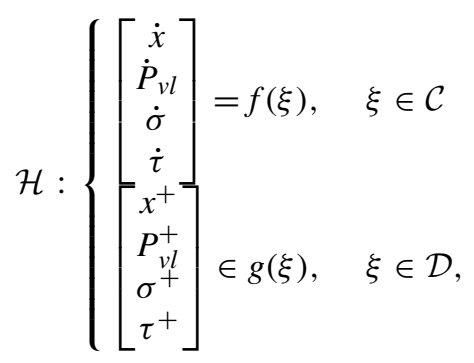

where $\xi=\left[\begin{array}{ll}x P_{v l} \sigma \tau & \tau\end{array}\right]^{\top} \in \mathbb{H}$ such that $\mathbb{H}:=\left[\mathrm{SOC}_{m}, \mathrm{SOC}_{M}\right] \times$ $\left[P_{m, 1}, P_{M, 1}\right] \times\left[P_{m, 2}, P_{M, 2}\right] \times \cdots \times\left[P_{m, \mathcal{I}}, P_{M, \mathcal{I}}\right] \times \mathcal{N} \times\{0,2 T\}$. Remember that $\sigma$ and $P_{v l}$ are the control inputs.
The maps $f$ and $g$ are

$$
\begin{aligned}
f(\xi):= & {\left[\begin{array}{c}
-\eta\left(P_{b a t}\right) \frac{\alpha P_{v l}+\gamma_{\sigma} P_{c l}-P_{g e n, k}}{C_{m a x}} \\
-K_{c} L(\alpha) P_{v l}-K_{c} \mathcal{L}\left(P_{v l}^{n s}-P_{k}^{*} 1_{\mathcal{I} \times 1}\right) \\
0_{N, 1} \\
r\left(\frac{\tau}{T}\right)
\end{array}\right], } \\
g(\xi):= & {\left[\begin{array}{c}
x \\
P_{v l} \\
\underset{j}{\operatorname{argmin}}\left(\frac{K}{\mathcal{T}(x, j)}-\beta \gamma_{j}\right) \\
0
\end{array}\right], }
\end{aligned}
$$

$x, P_{v l}$ and $P_{v l}^{n s}$ follow the definitions given in (5)-(9). $r\left(\frac{\tau}{T}\right)=\min \left(1,2-\frac{\tau}{T}\right)$ induces a minimum dwell time, defined by the positive parameter, $T$. In this way, a Zeno behavior is prevented. $K_{c}=\operatorname{diag}\left\{K_{c, i}, K_{c, 2}, \ldots, K_{c, \mathcal{I}}\right\}$, where $K_{c, i}>0$ defines the convergence speed of each $P_{v l, i}$, to a consensus objective between the VPLs. Likewise, $\mathcal{L}:=$ $\operatorname{diag}\left\{\ell_{1}, \ell_{2}, \ldots, \ell_{\mathcal{I}}\right\}$, where $\ell_{i} \in\{0,1\}$, which means that if $\ell_{i}=1$, the $i$-VPL is a leader and if $\ell_{i}=0$, the $i$-VPL is a follower. Moreover, $P_{k}^{*}\left(P_{v l}^{s}, \sigma, P_{g e n, k}\right)=\frac{P_{g e n, k}-\gamma_{\sigma} P_{c l}-\alpha P_{v l}^{s}}{\alpha^{s} \alpha^{\top}}>0$, with

$$
\begin{gathered}
\alpha^{s}:=\left[\alpha_{1} \operatorname{sw}\left(P_{v l, 1}, P_{m, 1}, P_{M, 1}\right), \alpha_{2} \operatorname{sw}\left(P_{v l, 2}, P_{m, 2}, P_{M, 2}\right), \ldots,\right. \\
\left.\alpha_{l} \operatorname{sw}\left(P_{v l, \mathcal{I}}, P_{m, \mathcal{I}}, P_{M, \mathcal{I}}\right)\right],
\end{gathered}
$$

denoting $\operatorname{sw}\left(s, s_{m}, s_{M}\right)=\max \left\{\left(s-s_{M}\right)\left(s-s_{m}\right), 0\right\}$, which switches the variable $s$ from 0 to 1 , when $s \notin\left[s_{m}, s_{M}\right]$, and reciprocally. Note that this is a binary vector variable that detects if $P_{v l, i}$ is saturated.

In this way, the leader $P_{v l, i}$ will enforce that $\alpha P_{v l}+\gamma_{\sigma} P_{c l}-$ $P_{g e n, k}$ goes to 0 . Note that the leader takes the information about the reference such that it is going to fulfil two objectives: ensure that the leader's VPL converges to the reference and reach a consensus with its neighbors. Referring to leader(s) selection, on one hand the user has to select the VPL(s) with maximum power and reliability, ensuring that it will be the last one to be saturated and it will not be disconnected. Indeed, if the leader is saturated, the other nodes, that has not saturated yet, will converge to this value, blocking them to achieve a larger power. On the other hand, each potential leader can present different transient times. However, this transient time is tuned by the parameters $K_{c, i}$. As $K_{c, i}$ is larger, the transient time will be shorter independently of the selected leader.

Assumption 1: The microgrid has been designed such that $\operatorname{rank}(\mathcal{L}) \leq 1$, i.e., at least, there is one leader, that ensures the convergence of $\alpha P_{v l}+\gamma_{\sigma} P_{c l}$ to $P_{g e n, k}$. Moreover, the leaders can not be disconnected and will be the last ones to be saturated.

Then, the so-called flow and jump sets are selected,

$$
\begin{aligned}
\mathcal{C} & :=\left\{\xi: J\left(x, \sigma^{+}\right) \geq J(x, \sigma)\right\} \cup\{\xi: \tau \in[0, T]\} \\
\mathcal{D} & :=\left\{\xi: J\left(x, \sigma^{+}\right) \leq J(x, \sigma)\right\} \cap\{\xi: \tau \in[T, 2 T]\} .
\end{aligned}
$$

This regularization is motivated by the fact that jumps cannot happen when the solution cannot improve the cost function $J$ after the jump, and the solution does not flow, at least, $T$ ordinary time after the last jump.

A solution to the item 2 of Problem 1 is included in the hybrid scheme (17)-(20). Note that the VPL dynamics are 


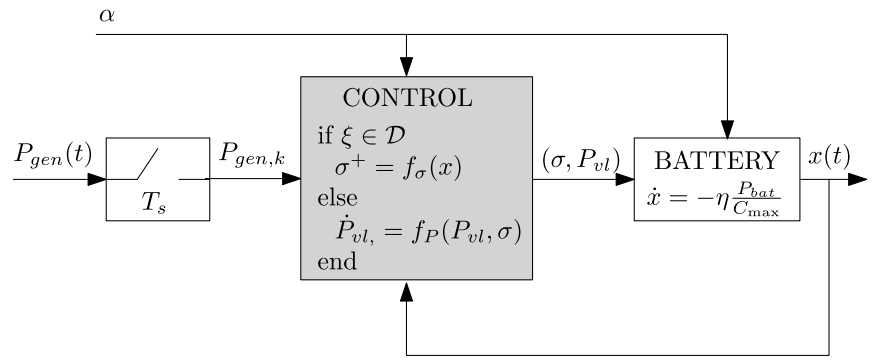

Fig. 1. Block diagram of the control mechanism, where $f_{\sigma}=$ $\underset{j}{\operatorname{argmin}}\left(\frac{K}{\mathcal{T}(x, j)}-\beta \gamma_{j}\right)$ and $f_{P}=-K_{c} L(\alpha) P_{v l}-K_{c} \mathcal{L}\left(\alpha P_{v l}+\gamma_{\sigma} P_{c l}-P_{g e n, k}\right)$.

defined in the map $f(\xi)$, by using the Laplacian matrix $L(\alpha)$, which takes into account the fact that a particular VPL is connected or disconnected. Moreover, matrix $\mathcal{L}$ gathers the information about the leader and follower character of each VPL. The controlled system implementation is depicted in Fig. 1.

Now, one can propose the following result for balancedSOC mode (see Definition 1).

Theorem 1: Consider a given $P_{m, i}, P_{M, i}>0$ for each $i$-VPL such that condition (3) and (4) are satisfied and any given $K_{c, i}>0$ and sampled time $T_{S}$ such that $\frac{1}{K_{c, i}}<T_{s}$ for all $i=1,2, . ., \mathcal{I}$. Likewise, the selected $\mathcal{L}$ fulfils Assumption 1. Moreover, for a given parameters $K_{x_{r}} \in(0,1]$, and $K>0$, the following holds for any initial condition $\xi(0,0) \in \mathbb{H}$ to system (17)-(20),

1) for all $(t, j) \in \operatorname{dom} \zeta$, the solutions $\xi(t, j)$ to (17)-(20) converge to $\mathcal{A}$.

2) All solutions to (17)-(20) eventually evolve continuously in the set $\mathcal{A} \cap\left\{\xi: x=x_{r, k}\right\}$.

Proof [Proof of item (i)]: The convergence proof cannot follow Lyapunov arguments, because the time to reach the desired attractor can take an arbitrarily long time, being non uniform. Due to this fact, the set $\mathcal{A}$ is unstable (even though globally attractive).

Without loss of generality, consider that the initial condition $\xi(0,0)$ flows in $\mathcal{C}$. Indeed, if the cost function is not susceptible to improve and the solution has not flowed a minimum-dwell time, $T$, the solution will remain evolving in the interior of $\mathcal{C}$. When a condition of $\mathcal{C}$ is violated that means that the solution will be in $\mathcal{D}$ and a jump will occur. After this jump, the system will evolve in $\mathcal{C}$, at least, for a minimum dwell time, i.e., until $\tau \geq T$. Note, that $\mathcal{A}$ is attractive, from the map $g(\xi)$. Indeed, the jumps of $\sigma$ ensure that for $x$ far from $x_{r, k}, x$ will approach slowly to it, and for $x$ close to $x_{r, k}$ and a fixed $\sigma^{+}, x$ will converge to $x_{r, k}$.

Proof of item (ii): In order to prove that $x$ converges to $x_{r, k}$ during flows, one needs to ensure that the solution in $\mathcal{C}$ guarantees that the power of each non-saturated VPL, $P_{v l, i}^{n s}$ for $i \in 1,2, \ldots, N$, will converge to a consensus value $P_{k}^{*}\left(P_{v l}^{s}, \sigma, P_{g e n, k}\right)=\frac{P_{g e n, k}-\gamma_{\sigma} P_{c l}-\alpha P_{v l}^{s}}{\alpha^{s} \alpha^{\top}}>0$. To this end, let us consider the following function

$$
\begin{aligned}
\left.V\left(P_{v l}^{n s}\right)\right)=\frac{1}{2}( & P_{v l}^{n s T} L\left(\alpha^{n s}\right) P_{v l}^{n s} \\
& \left.+\left(P_{v l}^{n s}-P_{k}^{*}\left(P^{s}\right) I_{\mathcal{I}}\right)^{\top} \mathcal{L}\left(P_{v l}^{n s}-P_{k}^{*} I_{\mathcal{I}}\right)\right),
\end{aligned}
$$

where $\alpha^{n s}:=\alpha-\alpha^{s}$ Hence, $L\left(\alpha^{n s}\right)$ represents the Laplacian matrix of the non saturated $P_{v l, i}, P_{v l, i}^{n s}$. From the definitions given in (7)-(9), it is hold $\dot{P}_{v l}=\dot{P}_{v l}^{n s}$. Moreover, it is easy to see that

$$
P_{v l}^{n s \top} L^{\top}\left(\alpha^{n s}\right) L(\alpha) P_{v l}=P_{v l}^{n s}{ }^{\top} L^{\top}\left(\alpha^{n s}\right) K_{c} L\left(\alpha^{n s}\right) P_{v l}^{n s} .
$$

Then, along flows:

$$
\begin{aligned}
\left\langle\nabla V_{c}\left(P_{v l}^{n s}\right)\right\rangle= & -P_{v l}^{n s \top} L^{\top}\left(\alpha^{n s}\right) K_{c} L\left(\alpha^{n s}\right) P_{v l}^{n s} \\
& -2 P_{v l}^{n s \top} L^{\top}\left(\alpha^{n s}\right) K_{c} \mathcal{L}\left(P_{v l}^{n s}-P_{k}^{*} I_{\mathcal{I}}\right) \\
& -\left(P_{v l}^{n s}-P_{k}^{*} I_{\mathcal{I}}\right)^{\top} \mathcal{L}^{\top} K_{c} \mathcal{L}\left(P_{v l}^{n s}-P_{k}^{*} I_{\mathcal{I}}\right) \leq 0 .
\end{aligned}
$$

The objective here is to guarantee a consensus for the nonsaturated powers of the VPLs, $P_{v l, i}^{n s} . K_{c, i}$ and $T_{s}$ are selected such that the response time of each $P_{v l, i}$ is shorter than $T_{s}$. Moreover, from the property of the Laplacian matrix, $\left\langle\nabla V_{c}\left(P_{v l}^{n s}\right)\right\rangle$ will be 0 , when all $P_{v l, i}^{n s}$ converge to $P_{k}^{*}=$ $\frac{P_{g e n, k}-\gamma_{\sigma} P_{c l}-\alpha_{i} P_{v i}^{s}}{\alpha^{s} \alpha^{\top}}$, given by the leader nodes, $i$ (defined by $\left.\ell_{i}=1\right)$. As the level sets of $V\left(P_{v l}^{n s}\right)$ are compact, the nonsmooth version of the LaSalle's invariance principle [24] can be applied, guaranteeing that each $P_{v l, i}^{n s}$ converges to the consensus $P_{k}^{*}$.

This last argument concludes the proof.

Theorem 1 proposes a controller, when the microgrid is in balanced-SOC mode. Nevertheless, a microgrid is susceptible of violating condition (10) and (11), requiring to switch to another controller designed for this situation.

\section{A. A Non-Balanced SOC Microgrid}

If condition (10) (and/or condition (11)) are violated, i.e., there is an excess (and/or lack) of generated power compared to the maximum (and/or minimum) demanded power capacity, the variable $\sigma$ is forced to be connected in open loop. That means, the battery will become empty (and/or full) and $\dot{x}$ cannot converge to zero. Unlike this control loop, the nonsaturated VPLs will be controlled following the consensus algorithm. Hence, one can define this hybrid system:

$$
\begin{aligned}
& \mathcal{H}_{s w}:\left\{\begin{array}{l}
{\left[\begin{array}{c}
\dot{x} \\
\dot{P}_{v l} \\
\dot{\sigma} \\
\dot{\tau}
\end{array}\right]=f_{s w}(\xi), \quad \xi \in \mathbb{H}} \\
{\left[\begin{array}{c}
x^{+} \\
P_{v l}^{+} \\
\sigma^{+} \\
\tau^{+}
\end{array}\right] \in g_{s w}(\xi), \quad \xi \in \emptyset,}
\end{array}\right. \\
& f_{s w}(\xi):=\left[\begin{array}{c}
-\eta\left(P_{b a t}\right) \frac{\alpha P_{v l}+\gamma_{\sigma} P_{c l}-P_{g e n, k}}{C_{m a x}} \\
-K_{c} L(\alpha) P_{v l}-K_{c} \mathcal{L}\left(P_{v l}^{n s}-P_{k}^{*} 1_{\mathcal{I} \times 1}\right) \\
0_{N, 1} \\
r\left(\frac{\tau}{T}\right)
\end{array}\right], \\
& g_{s w}(\xi):=\left[\begin{array}{llll}
x & P_{c l} & \sigma & 0
\end{array}\right]^{\top}, \\
& \left\{\begin{array}{lll}
\sigma=1_{1, N}, & \text { if } & 1_{1, N} P_{c l}+\sum_{i=0}^{\mathcal{I}} P_{M, i}<P_{\text {gen }, k} \\
\sigma=0_{1, N}, & \text { if } & 0_{1, N} P_{c l}+\sum_{i=0}^{\mathcal{I}} P_{m, i}>P_{\text {gen }, k} .
\end{array}\right.
\end{aligned}
$$

Theorem 2: Consider a given $P_{m, i}, P_{M, i}>0$ for each $i$-VPL such that condition (10) and/or (11) are not satisfied and any given $K_{c, i}>0$ and sampled time $T_{s}$ such that 
TABLE I

"HYLAB" DATA

\begin{tabular}{|l|l|}
\hline Device & Nominal value \\
\hline Programmable power supply & $6 \mathrm{~kW}$ \\
Photovoltaic field & $4 \mathrm{~kW}$ \\
Electronic load & $2 \mathrm{~kW}$ \\
Electrolyzer & $0.23 \mathrm{Nm}^{3} / h$ at $3 \mathrm{~kW}$ \\
Metal hydride tank & $7 \mathrm{Nm}^{3}$ at $5 \mathrm{bar}$ \\
PEMFC & $1.5 \mathrm{~kW}$ \\
Lead-acid battery bank capacity & $370 \mathrm{Ah}$ \\
Lithium battery bank capacity & $400 \mathrm{Ah}$ \\
\hline
\end{tabular}

$\frac{1}{K_{c, i}}<T_{s}$ for all $i=1,2, \ldots, \mathcal{I}$. Likewise, the selected $\mathcal{L}$ fulfils Assumption 1. Then, all solutions to (21)-(23) uniformly asymptotically converge to the set $\left\{\xi: x=x_{r, k}\right\}$.

Proof: Note that (21)-(23) is not a hybrid dynamical system, but a continuous-time dynamical system, because the jump set is empty. In this functioning mode, the system can provide uniform asymptotic stability of $\left\{\xi: x=x_{r, k}\right\}$. The proof follows the arguments given in item (ii) of Theorem 1, with either $\gamma_{\sigma}=1_{1 \times N}$ if condition (10) is not satisfied, or $\gamma_{\sigma}=0_{1 \times N}$ if condition (11) is not satisfied.

\section{B. Guideline for Parameter Selection}

In the HDS scheme proposed above there are some parameters to be tuned to adjust the reference, $x_{r, k},\left(K_{x, k}\right)$, the CPLs $(K)$ and the CVLs $\left(K_{c}\right)$. We summarize here the rules to adjust these parameters.

- $K_{x, r} \in(0,1]$ : This parameter allows to modulate the varying behavior of the reference, $x_{r, k}$. If the user desires that the reference presents a larger varying behavior, then $K_{x, r}$ has to be closer to 1 . However, if the user prefers a more static behavior of the reference, then $K_{x, r}$ has to be closer to 0 .

- $K>0$ : This parameter manages the number of possible connected CPLs. If the user requires to increase the number of connected CPLs, then $K$ has to be larger.

- $K_{c}>0$ : This parameter drives the convergence speed of the CVLs to a consensus. Indeed, if the user needs that this convergence is fast, he/she has to chose larger $K_{c, i}$.

Note that the stability and robustness properties given in the paper are guaranteed for all these possible combinations.

\section{EXPERIMENTAL RESUlTS}

This section is devoted to validate the proposed control algorithm. The experiments are performed using a detailed dynamic model of a microgrid located at the University of Seville [25]. This microgrid is composed of a lead-acid battery bank, a lithium-ion battery bank, a photovoltaic field, an electrolizer, a fuel cell, hydrogen storage by means of two hydride tanks, $N=7$ CPLs and $\mathcal{I}=7$ CVLs (emulated by the electronic load) and several DC/DC converters. The specifications of the different devices are described in Table I and Figure 2 shows a picture of the microgrid. The methodology for DSM is tested in this microgrid without using the hydrogen storage elements and with the parameters given in Table II in two different situations, depending on generation: a sunny and

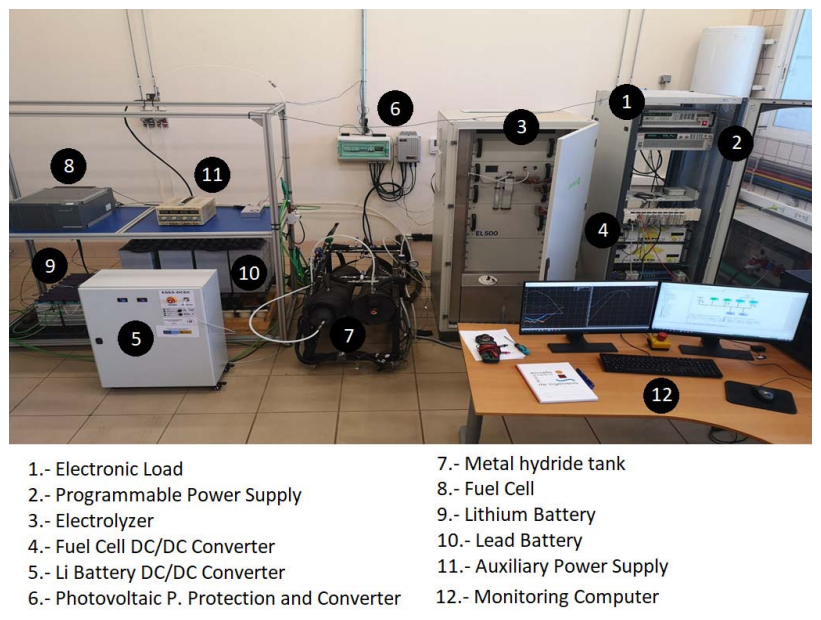

Fig. 2. "Hylab" microgrid.

TABLE II

PARAMETERS FOR DR

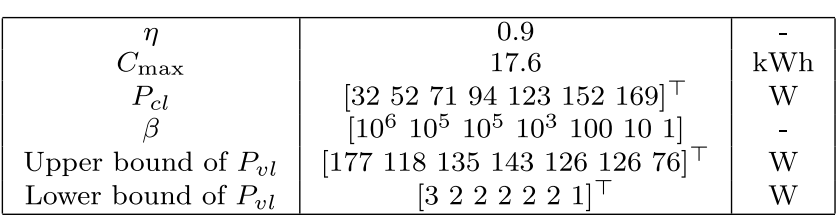

TABLE III

SELECTED PARAMETERS

\begin{tabular}{|c|c|c|}
\hline $\mathrm{SOC}_{M}$ & 90 & $\%$ \\
\hline $\mathrm{SOC}_{m}$ & 10 & $\%$ \\
\hline$K_{x_{r}}$ & 1 & - \\
\hline $\mathcal{L}$ & {$\left[\begin{array}{lllllll}1 & 0 & 0 & 0 & 0 & 0 & 0\end{array}\right]$} & - \\
\hline$T$ & 1200 & $\mathrm{~s}$ \\
\hline$T_{s}$ & 1 & $\mathrm{~s}$ \\
\hline$K$ & 10 & - \\
\hline$K_{c}$ & $10 I_{7}$ & $\mathrm{~W}$ \\
\hline
\end{tabular}

a cloudy day. The control algorithm must behave in a different way depending on the available solar power.

The selected parameters for the controlled microgrid appear in Table III. Moreover, we select $K_{x_{r}}=1$, which means that the chosen reference follows the dynamic of the sampled $P_{g e n, k}$ and a leader-follower consensus algorithm with 1 leader with the following Laplacian matrix:

$$
L(\alpha)=\alpha^{\top}\left[\begin{array}{ccccccc}
2 & -1 & 0 & 0 & 0 & 0 & -1 \\
-1 & 2 & -1 & 0 & 0 & 0 & 0 \\
0 & -1 & 2 & -1 & 0 & 0 & 0 \\
0 & 0 & -1 & 2 & -1 & 0 & 0 \\
0 & 0 & 0 & -1 & 2 & -1 & 0 \\
0 & 0 & 0 & 0 & -1 & 2 & -1 \\
-1 & 0 & 0 & 0 & 0 & -1 & 2
\end{array}\right] \alpha
$$

The microgrid will work with the hybrid control proposed in (17)-(20), when we have a balanced-SOC mode, that means conditions (10) and (11) are fulfilled. On the contrary, the system will be controlled with the algorithm given in (21)-(23), which manages only $P_{v l, i}^{n s}$, because in this case all (or any) CPLs must be connected to the microgrid. 


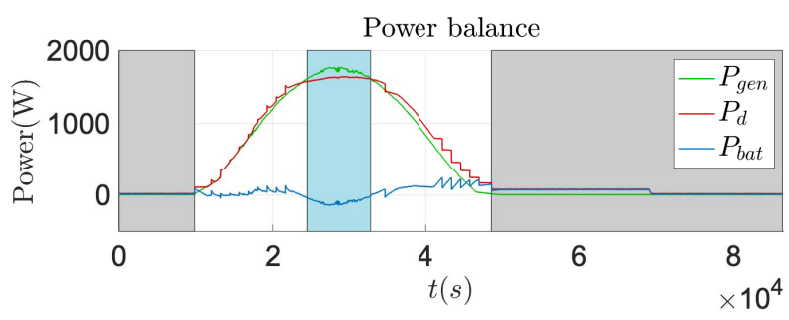

Fig. 3. Power balance, showing the generated Power, $P_{\text {gen }}$, in green, the demanded power, $P_{d}$ in red and the battery power, $P_{b a t}$ in blue, in a sunny day. The shaded area represents the period when condition (10) (blue shadow) and (11) (grey shadow) are not fulfilled.

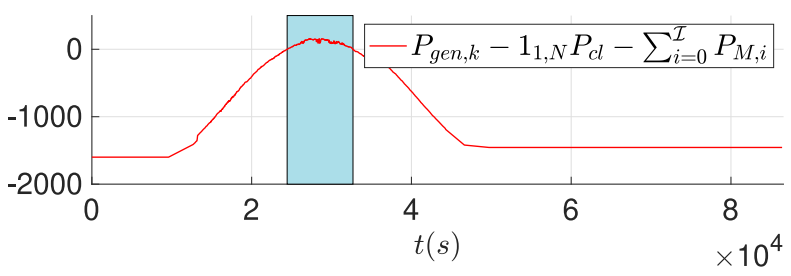

Fig. 4. Evolution of $P_{g e n, k}-1_{1, N} P_{c l}-\sum_{i=0}^{\mathcal{I}} P_{M, i}$. The shaded area represents the period when condition (10) is not fulfilled.

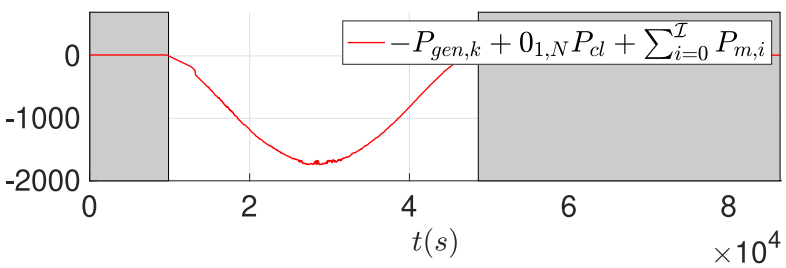

Fig. 5. Evolution of $P_{g e n, k}-0_{1, N} P_{c l}-\sum_{i=0}^{\mathcal{I}} P_{M, i}$. The shaded area represents the period when condition $(11)$ is not fulfilled.

After some $P_{g e n}(t)$ evolutions, it is possible to characterize a $P_{g e n, k}$ which evolves in the following slot

$$
0 \mathrm{~W} \leq P_{\text {gen }, k} \leq 3500 \mathrm{~W},
$$

such that, $P_{\text {gen }, a v}^{e s}=1750 \mathrm{~W}$ and $P_{\text {gen,amp }}^{e s}=1750 \mathrm{~W}$.

\section{A. Sunny Day}

First, a data base of $P_{g e n}(t)$ in a sunny day is taken, during a complete day (86400 seconds). As mentioned above, the proposed hybrid control will manage the microgrid, whereas the demanded power is larger than the generated one. Hence, Fig. 3 displays the $P_{g e n}(t), P_{d}(\sigma, t)$ and $P_{b a t}(\sigma, t)$ evolutions. It is worth noting that while condition (10) is satisfied, $P_{d}(\sigma, t)$ converges to $P_{\text {gen }}(t)$. Note that Figs. 4 and 5 show the validation of condition (10) and condition (11), respectively. If these ones are positive, it means that condition (10) and (11) are not satisfied, which is highlighted with a shaded area. Likewise, during the slot time that condition (10) and (11) are violated, the microgrid is controlled by the algorithm given in (21)-(23). Note that an excess of energy is stocked in the battery, leading to a negative $P_{b a t}$, as shown in the figure.

Figure 7 and Fig. 8 show the CPLs and VPLs evolutions, respectively. On one hand, the picture draws the switching of the CPLs, according to the control input, $\sigma$. Likewise, note that these CPLs are connected and disconnected into the microgrid,
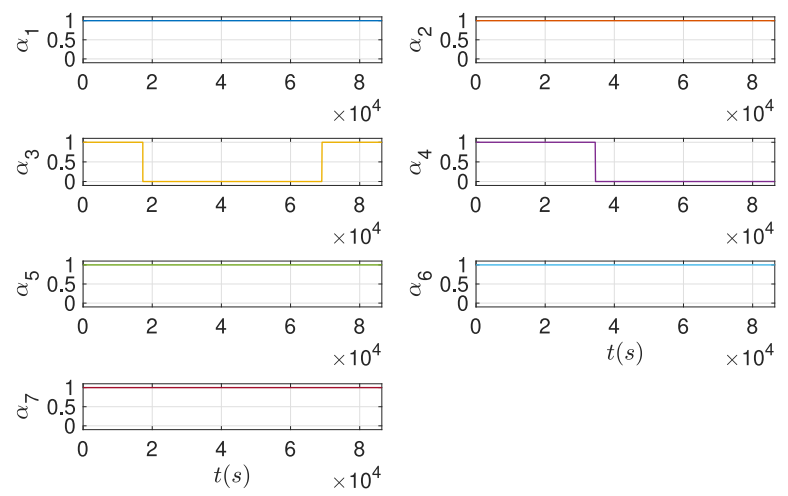

Fig. 6. $\alpha$ scenario.

governed by the priority levels, $\beta$. During the slot time when condition (10) or (11) are not fulfilled, all loads are supplied or any load is connected, respectively. On the other hand, the non-saturated VPLs evolve in consensus with an $\alpha$ scenario given in Fig. 6, where $\alpha_{4}$ represents that $\mathrm{VPL}_{4}$ is disconnected and that $\mathrm{VPL}_{3}$ turns to be a connected constant load, during a slot time. Hence, the non-saturated VPLs achieves the discrete variable $P_{k}^{*}$ such that $\left|P_{d}-P_{g}\right|$ goes to zero, guaranteeing robustness with respect to plug and play. In particular, when any VPL is disconnected $\left(\alpha_{i}=0\right)$, as $\mathrm{VPL}_{4}$ with $P_{v l, 4}$ at $34560 \mathrm{~s}$, when any $P_{v l, i}$ turns to be constant, as VPL 3 with $P_{v l, 3}$ during the slot time $[17280,69120] \mathrm{s}$ or when any $P_{v l, i}$ saturates in its upper bound, as occurs for $P_{v l, 7}$ at $17769 \mathrm{~s}$, $P_{v l, 2}$ at $21990 \mathrm{~s}, P_{v l, 6}$ and $P_{v l, 5}$ at $22725 \mathrm{~s}, P_{v l, 4}$ at 24266 s, and $P_{v l, 4}$ at $24266 \mathrm{~s}$. Note that Assumption 1 is satisfied, i.e., the VPLs evolve in consensus, getting their upper bound, being the leader $P_{v l, 1}$ the last one to saturate.

Figure 7 and Fig. 8 show the CPLs and VPLs evolutions, respectively. On one hand, the picture draws the switching of the CPLs, according to the control input, $\sigma$. Likewise, note that these CPLs are connected and disconnected into the microgrid, governed by the priority levels, $\beta$. During the slot time when condition (10) or (11) are not fulfilled, all loads are supplied or any load is connected, respectively. On the other hand, the non-saturated VPLs evolve in consensus with an $\alpha$ scenario given in Fig. 6, where $\alpha_{4}$ represents that $\mathrm{VPL}_{4}$ is disconnected and that $\mathrm{VPL}_{3}$ turns to be a connected constant load, during a slot time. Hence, the non-saturated VPLs achieves the discrete variable $P_{k}^{*}$ such that $\left|P_{d}-P_{g}\right|$ goes to zero, guaranteeing robustness with respect to plug and play. In particular, when any VPL is disconnected $\left(\alpha_{i}=0\right)$, as VPL 4 with $P_{v l, 4}$ at $34560 \mathrm{~s}$, when any $P_{v l, i}$ turns to be constant, as $\mathrm{VPL}_{3}$ with $P_{v l, 3}$ during the slot time $[17280,69120] \mathrm{s}$ or when any $P_{v l, i}$ saturates in its upper bound, as occurs for $P_{v l, 7}$ at $17769 \mathrm{~s}$, $P_{v l, 2}$ at $21990 \mathrm{~s}, P_{v l, 6}$ and $P_{v l, 5}$ at $22725 \mathrm{~s}, P_{v l, 4}$ at 24266 s, and $P_{v l, 4}$ at $24266 \mathrm{~s}$. Note that Assumption 1 is satisfied, i.e., the VPLs evolve in consensus, getting their upper bound, being the leader $P_{v l, 1}$ the last one to saturate.

Finally, the SOC evolution is shown in Fig. 9. Note that, in the initial condition, the SOC is in $80 \%$, the CPLs are disconnected and the VPLs are in its minimum values $0 \mathrm{~W}$. Indeed, at this initial condition the microgrid is controlled with 


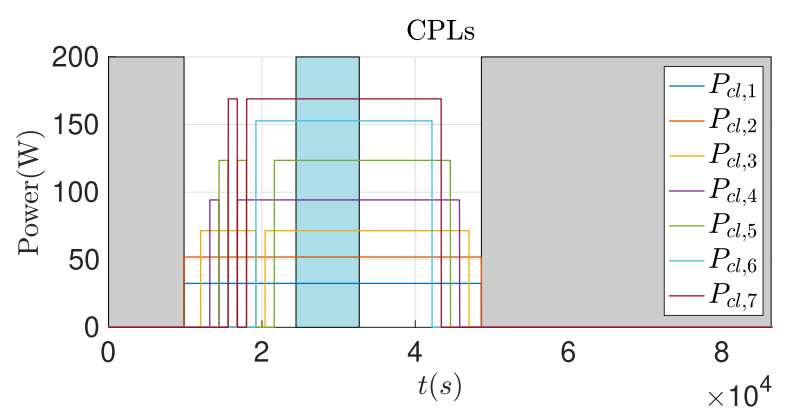

Fig. 7. Power evolutions of the CPLs in a sunny day. The shaded area in blue and grey represent the period when condition (10) and (11), respectively, are not fulfilled.

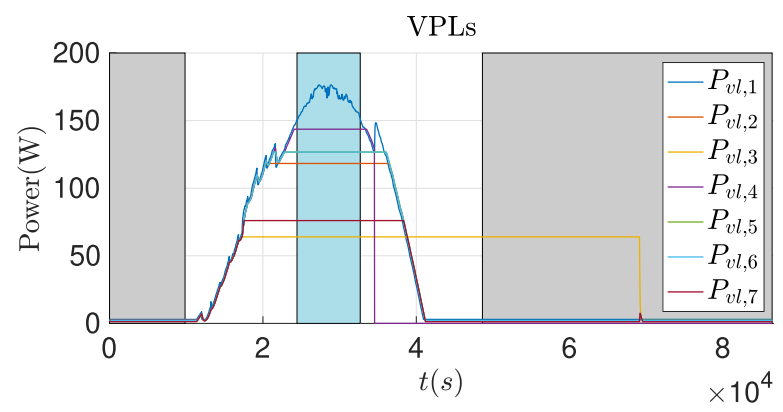

Fig. 8. Power evolutions of the CVLs in a sunny day. The shaded area in blue and grey represent the period when condition (10) and (11), respectively, are not fulfilled.

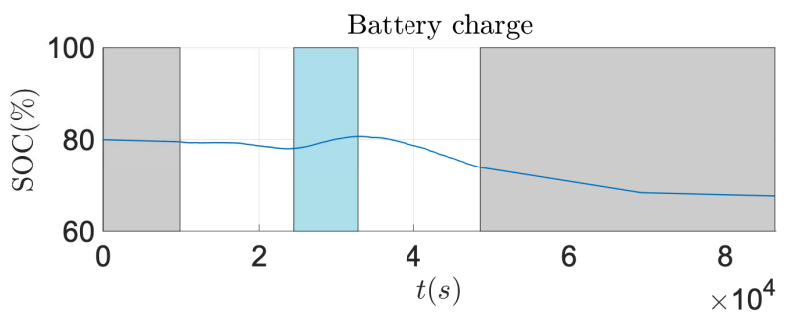

Fig. 9. SOC evolution in a sunny day. The shaded area in blue and grey represent the period when condition (10) and (11), respectively, are not fulfilled.

the hybrid control algorithm given in (21)-(23). When, condition (11) is fulfilled, the CPLs start connecting, reducing the SOC. Then, the SOC evolves following the tendency of $P_{\text {gen }}$, i.e., if $P_{\text {gen }}$ increases, the SOC also goes up, and reciprocally. Let us highlight that the SOC achieves its maximum rank, when condition (10) is not satisfied any more.

\section{B. Cloudy Day}

Now, the proposed control is tested in an unfavourable situation, which is a cloudy day. In this simulation, one can consider the same scenario as in the sunny day, including the $\alpha$ evolutions, i.e., a disconnection of $\mathrm{VPL}_{4}$ and a change of $\mathrm{VPL}_{3}$ to a constant power load during a time slot, according to Fig. 6. In Fig. 10, the important oscillations of $P_{\text {gen }}$ are appreciated besides not violating condition (10), as is shown in Fig. 11. Nevertheless, condition (11) is not satisfied during the slot time patched in the figure. The evolution of condition (11)

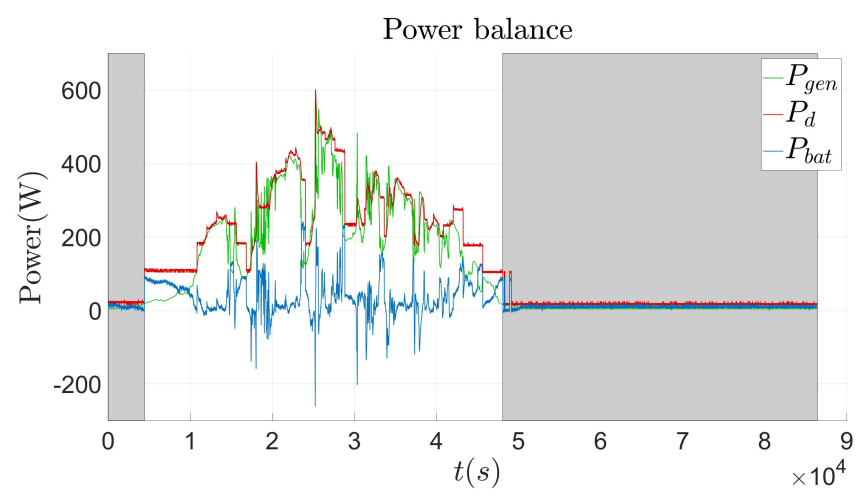

Fig. 10. Power balance, showing the generated Power, $P_{\text {gen }}$, in green, the demanded power, $P_{d}$ in red and the battery power, $P_{b a t}$ in blue, in a cloudy day. The shaded area represents the period when condition (11) is not fulfilled.

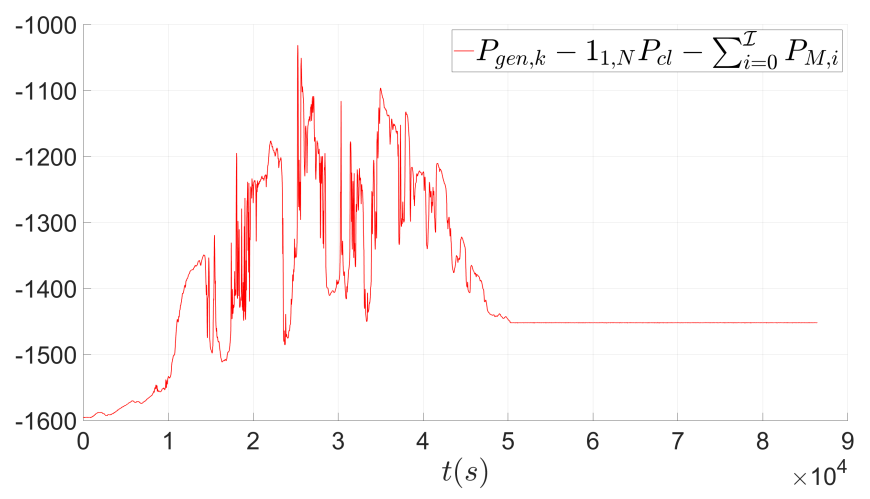

Fig. 11. Evolution of $P_{g e n, k}-1_{1, N} P_{c l}-\sum_{i=0}^{\mathcal{I}} P_{M, i}$.

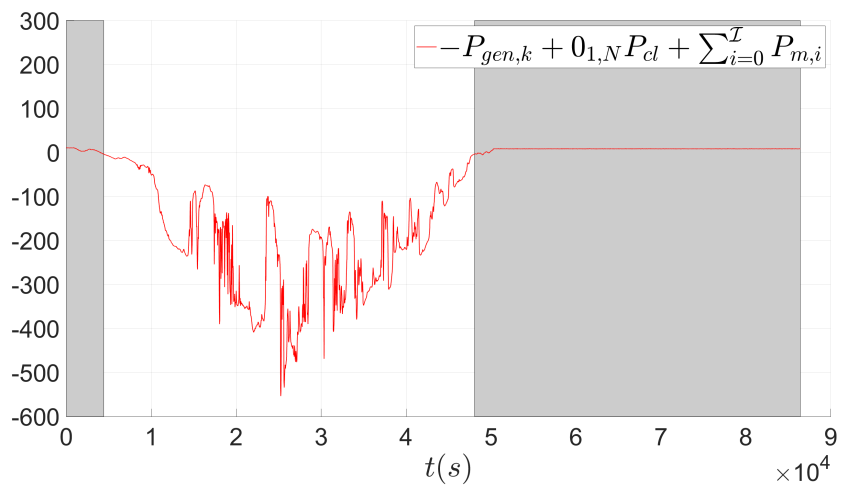

Fig. 12. Evolution of $P_{g e n, k}-0_{1, N} P_{c l}-\sum_{i=0}^{\mathcal{I}} P_{M, i}$. The shaded area represents the period when condition (11) is not fulfilled.

is shown in Fig. 12. It is worth noting that $P_{d}$ converges to $P_{g e n}$ even in this strong perturbed situation.

Figures 13 and 14 show the CPL and VPL evolutions, respectively. Contrary to a sunny day, there are more switching in the less priority loads. Likewise, the VPLs evolve with strong oscillations, following $P_{g e n, k}$. In this scenario, there is 


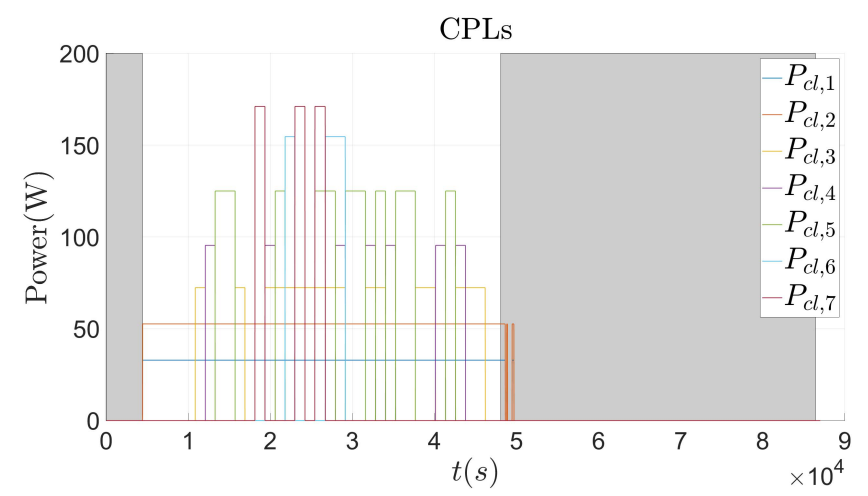

Fig. 13. Power evolutions of the CPLs in a cloudy day. The shaded area represents the period when condition (11) is not fulfilled.

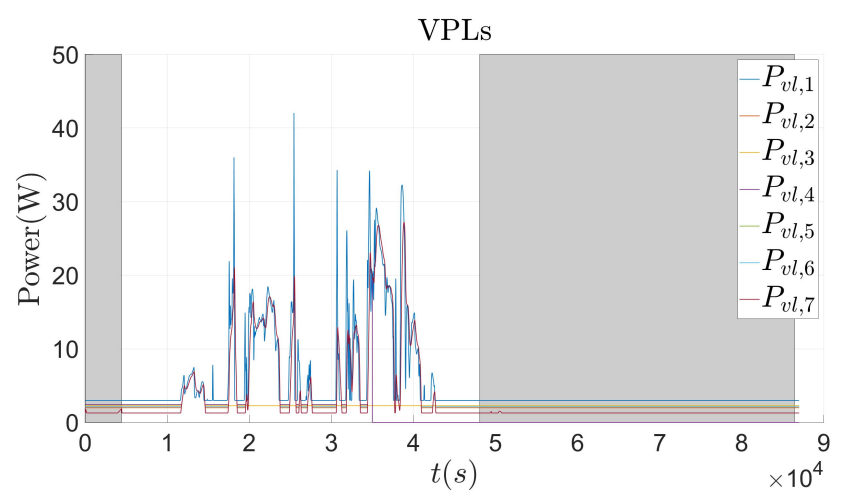

Fig. 14. Power evolutions of the CVLs in a cloudy day. The shaded area represents the period when condition (11) is not fulfilled.

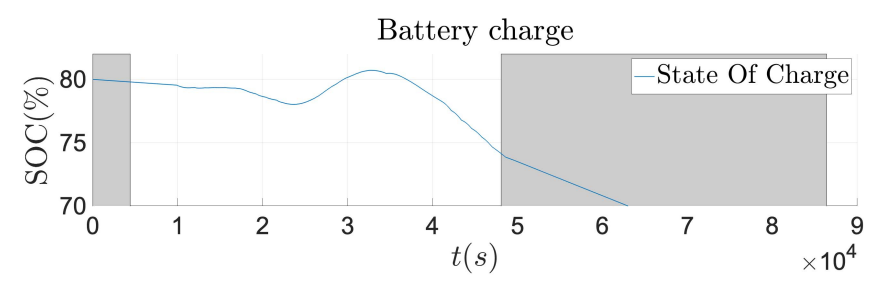

Fig. 15. SOC evolution in a cloudy day. The shaded area represents the period when condition (11) is not fulfilled.

not a VPL that achieves its upper bound. Finally, it is natural to expect a decreasing SOC in this situation, as shown in Fig. 15.

These simulations validate Theorem 1 and Theorem 2 which propose the controllers for a DR, getting a robust and scalable islanded microgrid.

\section{CONCLUSION}

In this paper, HDS and MAS theory are used to propose a robust hybrid control to manage the DR in islanded microgrids. In particular, the demand presents shifting loads (CPLs) as well as adjusting loads (VPLs). These theories are appropriated here because both: the system is composed of hybrid dynamic and a consensus between the adjusting loads is desirable to achieve suited performance. A non-uniform convergence of the SOC to the reference is ensured and robustness properties with respect to plug and play and parameter variations is guaranteed.

The result is tested in a microgrid existing at the University of Seville, "Hylab". Two scenarios are shown, a sunny day and, one more unfavourable, a cloudy day. This perform provides an appropriated SOC evolution, when there is more generation than demand as well as the opposite case.

In future work, a distributed solution without considering a leader-follower consensus algorithm will be considered as well as the reactive power management in AC microgrids.

\section{REFERENCES}

[1] R. H. Lasseter, "Microgrids," in Proc. IEEE Power Eng. Soc. Winter Meeting, vol. 1, 2002, pp. 305-308.

[2] D. E. Olivares et al., "Trends in microgrid control," IEEE Trans. Smart Grid, vol. 5, no. 4, pp. 1905-1919, Jul. 2014.

[3] P. Palensky and D. Dietrich, "Demand side management: Demand response, intelligent energy systems, and smart loads," IEEE Trans. Ind. Informat., vol. 7, no. 3, pp. 381-388, Aug. 2011.

[4] K. Kostkova, L. Omelina, P. Kycina, and P. Jamrich, "An introduction to load management," Elect. Power Syst. Res., vol. 95, pp. 184-191, Feb. 2013.

[5] T. Logenthiran, D. Srinivasan, and T. Z. Shun, "Demand side management in smart grid using heuristic optimization," IEEE Trans. Smart Grid, vol. 3, no. 3, pp. 1244-1252, Sep. 2012.

[6] M. Alizadeh, X. Li, Z. Wang, A. Scaglione, and R. Melton, "Demandside management in the smart grid: Information processing for the power switch," IEEE Signal Process. Mag., vol. 29, no. 5, pp. 55-67, Sep. 2012.

[7] D. Li, W. Y. Chiu, and H. Sun," Microgrid. Advanced Control Methods and Renewable Energy System Integration. Boston, MA, USA: Elsevier, 2017, ch. Demand Side Management in Microgrid Control Systems.

[8] V. Jayadev and K. S. Swarup, "Optimization of microgrid with demand side management using genetic algorithm," in Proc. IET Conf. Power Unity Whole Syst. Approach, 2013, p. 1.12.

[9] Z. Chen, L. Wu, and Y. Fu, "Real-time price-based demand response management for residential appliances via stochastic optimization and robust optimization," IEEE Trans. Smart Grid, vol. 3, no. 4 pp. 1822-1831, Dec. 2012.

[10] C. Bordons, F. Garcia-Torres, and M. A. Ridao, Model Predictive Control of Microgrids. Cham, Switzerland: Springer Nat., 2020.

[11] K. Miyazaki, K. Kobayashi, S.-I. Azuma, N. Yamaguchi, and Y. Yamashita, "Design and value evaluation of demand response based on model predictive control," IEEE Trans. Ind. Informat., vol. 15, no. 8, pp. 4809-4818, Aug. 2019.

[12] V. A. Freire, L. V. R. De Arruda, C. Bordons, and J. J. Márquez, "Optimal demand response management of a residential microgrid using model predictive control," IEEE Access, vol. 8, pp. 228264-228276, 2020.

[13] R. Goebel and R. G. Sanfelice, and A. R. Teel, Hybrid Dynamical Systems: Modeling, Stability, and Robustness. Princeton, NJ, USA: Princeton Univ. Press, 2012.

[14] R. Olfati-Saber and R. M. Murray, "Consensus problems in networks of agents with switching topology and time-delays," IEEE Trans. Autom. Control, vol. 49, no. 9, pp. 1520-1533, Sep. 2004.

[15] D. V. Dimarogonas, E. Frazzoli, and K. H. Johansson, "Distributed event-triggered control for multi-agent systems," IEEE Trans. Autom. Control, vol. 57, no. 5, pp. 1291-1297, May 2012.

[16] J. P. Torreglosa, P. García, L. M. Fernández, and F. Jurado, "Hierarchical energy management system for stand-alone hybrid system based on generation costs and cascade control," Energy Convers. Manage., vol. 77, pp. 514-526, Jan. 2014.

[17] J. Lagorse, M. G. Simões, and A. Miraoui, "A multiagent fuzzy-logicbased energy management of hybrid systems," IEEE Trans. Ind. Appl., vol. 45, no. 6, pp. 2123-2129, Nov./Dec. 2009.

[18] C. Albea-Sanchez, "Hybrid dynamical control based on consensus algorithms for current sharing in DC-bus microgrids," Nonlinear Anal. Hybrid Syst., vol. 39, Feb. 2021, Art. no. 100972.

[19] T. H. Chang, A. Nedić, and A. Scaglione, "Distributed constrained optimization by consensus-based primal-dual perturbation method," IEEE Trans. Autom. Control, vol. 6, no. 59, pp. 1524-1538, Jun. 2014. 
[20] N. Rahbari-Asr and M.-Y. Chow, "Cooperative distributed demand management for community charging of PHEV/PEVs based on KKT conditions and consensus networks," IEEE Trans. Ind. Informat., vol. 3, no. 10, pp. 1907-1916, Aug. 2014.

[21] W. Zhang, Y. Xu, W. Liu, F. Ferrese, and L. Liu, "Fully distributed coordination of multiple DFIGs in a microgrid for load sharing," IEEE Trans. Smart Grid, vol. 4, no. 2, pp. 806-815, Jun. 2013.

[22] A. S. Hintz, U. R. Prasanna, and K. Rajashekara, "Hybrid multi-agent based resilient control for EV connected micro grid system," in Proc. IEEE Transp. Electrif. Conf. Expo (ITEC), Dearborn, MI, USA, 2014, pp. 1-6.

[23] R. Olfati-Saber and R. M. Murray, "Graph rigidity and distributed formation stabilization of multi-vehicle systems," in Proc. IEEE Conf. Decis. Control, vol. 3. Las Vegas, NV, USA, 2002, pp. 2965-2971.

[24] D. Shevitz and B. Paden, "Lyapunov stability theory of nonsmooth systems," IEEE Trans. Autom. Control, vol. 39, no. 9, pp. 1910-1914, Sep. 1994.

[25] L. Valverde, F. Rosa, C. Bordons, and J. Guerra, "Energy management strategies in hydrogen smart-grids: A laboratory experience," Int. J. Hydrogen Energy, vol. 41, no. 31, 2016, Art. no. 13715.

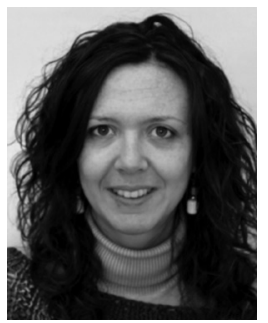

Carolina Albea received the Ph.D. degree in automatic control from the University of Sevilla, Spain, and the University of Grenoble, France, in 2010. From 2010 to 2011, she held a postdoctoral position with CEA-LETI (Minatec Campus), Grenoble, France, on the control of nanoelectronic circuits. From 2011 to 2020, she became an Associate Professor with the University of Toulouse III (Université Paul Sabatier) and her research was performed with the LAAS-CNRS. In 2020, she joined the University of Seville, Spain. Her topics of interest cover hybrid control of switched systems, hybrid dynamical systems application, distributed control, and control of electronic converters.

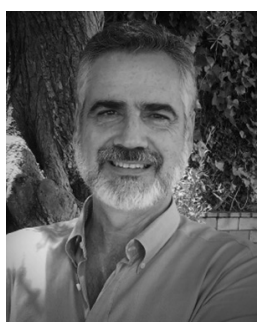

Carlos Bordons (Senior Member, IEEE) received the Ph.D. degree in electrical engineering in 1994. $\mathrm{He}$ joined the Escuela Superior de Ingenieros of Seville, Spain, as an Assistant Professor, where he is currently a Full Professor of Systems Engineering and Automatic Control. His current research interests include advanced process control, especially model predictive control and its application to microgrids and hybrid vehicles. He is currently a member of the Governing Board of Corporacón Tecnológica de Andaluía and the Head of the Laboratory of Engineering for Energy and Environmental Sustainability.

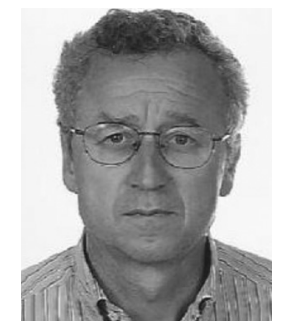

Miguel A. Ridao (Member, IEEE) received the $\mathrm{Ph} . \mathrm{D}$. degree in industrial engineering from the University of Seville, Spain, in 1995, where he is a Professor of System Engineering and Automation with the Engineering School. His current research interests include distributed control, control of water systems, hybrid vehicles, automation of supply channels, and decision support systems. 